\title{
Rapid Proteomic Screen of CRISPR Experiment Outcome by Data Independent Acquisition Mass Spectrometry: A Case Study for HMGN1
}

\author{
Martin Mehnert ${ }^{1,5}$, Wenxue $\mathrm{Li}^{2,5}$, Chongde $\mathrm{Wu}^{2}$, Barbora Salovska ${ }^{2,3}$, Yansheng Liu ${ }^{2,4 *}$
}

1. Department of Biology, Institute of Molecular Systems Biology, ETH Zurich, Zurich, Switzerland

2. Yale Cancer Biology Institute, Yale University, West Haven, CT 06516, USA

3. Department of Genome Integrity, Institute of Molecular Genetics of the Czech Academy of Sciences, Prague, Czech Republic

4. Department of Pharmacology, Yale University School of Medicine, New Haven, CT 06520, USA

5. These two authors contribute equally to the study.

* Corresponding author:

Yansheng Liu yansheng.liu@yale.edu

Assistant Professor

Department of Pharmacology

Yale University School of Medicine

\begin{abstract}
CRISPR-Cas gene editing holds substantial promise in many biomedical disciplines and basic research. Due to the important functional implications of non-histone chromosomal protein HMG-14 (HMGN1) in regulating chromatin structure and tumor immunity, we performed gene knockout of HMGN1 by CRISPR in cancer cells and studied the following proteomic regulation events. In particular, we utilized DIA mass spectrometry (DIA-MS) and reproducibly measured more than 6200 proteins (protein- FDR 1\%) and more than 82,000 peptide precursors in the single MS shots of two hours. HMGN1 protein deletion was confidently verified by DIA-MS in all of the clone- and dish- replicates following CRISPR. Statistical analysis revealed 147 proteins changed their expressions significantly after HMGN1 knockout. Functional annotation and enrichment analysis indicate the deletion of HMGN1 induces the histone inactivation, various stress pathways, remodeling of extracellular proteomes, cell proliferation, as well as immune regulation processes such as complement and coagulation cascade and interferon alpha/ gamma response in cancer cells. These results shed new lights on the cellular functions of HMGN1. We suggest that DIA-MS can be reliably used as a rapid, robust, and cost-effective proteomicscreening tool to assess the outcome of the CRISPR experiments.
\end{abstract}




\section{Introduction}

2 The clustered regularly interspaced short palindromic repeat (CRISPR) genome-editing system

3 was demonstrated to be a revolutionary tool in many biological and biomedical fields, including

4 studying gene functions, creating new cell and animal models, developing therapeutic agents,

5 and potentially to treat diseases. CRISPR-Cas9 was adapted from a naturally occurring genome

6 editing system in prokaryotic organisms that provides a form of acquired immunity [1]. In this

7 system, CRISPR sequences are DNA fragments from viruses that have previously infected the

8 prokaryote and are used to detect and destroy DNA from similar viruses during subsequent

9 infections, whereas Cas9 (or "CRISPR-associated 9") is an enzyme that cuts foreign DNA.

10 Usually, the Cas9 nuclease (or other enzymes) can be delivered with a synthetic guide RNA

11 (gRNA) into a cell so that the cell's genome can be cut at a desired location [2-6]. Following

12 DNA cleavage, researchers can use the eukaryotic cell's own repair systems to either fuse broken

13 chromosomal ends together via non-homologous end joining (NHEJ) or, in the presence of donor

14 DNA, introduce exogenous sequence via homology-directed repair (HDR), for the purpose of

15 gene knockout or knock in respectively [2-5].

17 A growing number of applications of CRISPR-Cas9 require a better understanding,

18 characterization, and management of CRISPR efficacy and off-target effects. Many prediction

19 tools and experimental approaches have been developed for these aims [7-10]. For example,

20 Cas9 nickase activity was used to significantly reduce off-target activity and the application of

21 paired guide RNAs (gRNAs) was shown to increase the efficiency of gene knockouts [11, 12].

22 Currently, the successful implementation of CRISPR experiment for a target gene or gene set is

23 routinely validated by PCR using primers flanking the deleted region at the DNA level or by

24 western blotting of the protein targets at the protein level. The next generation sequencing (NGS)

25 techniques using e.g., amplicon-based kits have been successfully applied for screening

26 CRISPR-Cas9 induced mutations, efficacy, and both on-target and off-target effects [13-16].

28 Due to the limitations of antibody such as cross-reactivity, the mass spectrometry (MS) based

29 methods such as targeted proteomics $[17,18]$ have been widely adopted to detect and quantify

30 proteins of interest. Over the last decade, the proteome has become precisely measurable. The

31 acute state of the proteome - the proteotype - is a useful indicator of the cellular state and 
1 important for studying disease phenotype, development, pharmacological responses, and many

2 other aspects in life science research [19, 20]. The last five years have witnessed significant

3 technological improvements in MS-based proteomics especially in proteome coverage and

4 measurement reproducibility of large sample cohorts. Particularly, data-independent acquisition

5 (DIA) methods, such as SWATH mass spectrometry (SWATH-MS) combined with targeted data

6 extraction has achieved unprecedented reproducibility, which are capable of generating

7 quantitative matrices for thousands of proteins measured across multiple samples [20-22].

8 Recently, SWATH-MS was proven to be reproducible in a cross-lab study for $>4000$ proteins

9 quantified in mammalian cells [23]. Moreover, the statistical strategies controlling the quality of

10 protein identification and quantification in large-scale DIA-MS have matured [24, 25]. Because

11 proteins catalyze and control directly most biological processes, and because proteotype

12 characterization essentially bridges the genotypic variation and phenotype diversity, we propose

13 that a sensitive, reproducible, robust, and rapid proteomic measurement enabled by e.g., DIA-MS

14 would provide a general and powerful alternative approach to assess the outcome of a CRISPR

15 experiment, to not only verify the successful knockout of the target gene (and its protein

16 expression), but also to profile perturbed signaling pathways and quantitative phenotypes.

18 Herein, we focused on an example of a human protein, non-histone chromosomal protein HMG-

1914 (High Mobility Group Nucleosome Binding Domain 1, or HMGN1). Others and we have

20 previously observed the HMGN1 mRNA and protein overexpression in Down Syndrome (DS,

21 trisomy 21), which supports the hypothesis that HMGN1 could increase the chromatin

22 accessibility and cause transcriptional dysregulation [26, 27]. Based on previous literatures,

23 HMGN1 is associated to two major processes, transcriptional regulation and tumor immunity

24 [28-36]. Despite of the emerging importance of HMGN1, there are currently no proteomic data

25 sets available to understand the proteotype induced by its exclusive dosage imbalance in cells. In

26 the present study we therefore analyzed the HMGN1 knockout cancer cells by DIA-MS to

27 further understand the functional roles of HMGN1.

\section{Results}

30 To specifically knock out HMGN1 by CRISPR, we transfected the human T-REx-HeLa CCL2

31 cells with two gRNAs that target the second and third exon of HMGN1, respectively. Single 
1 cells with positive GFP signal indicating Cas9 and gRNA expression were then sorted and

2 expanded. To investigate the proteomic variation between these cell clones, three of them

3 (named Clone A, B, and C hereafter) were verified by western blot to be negative with HMGN1

4 antibody staining, as compared to the wildtype control cells (Figure 1, left panel), and included

5 in the study as clone- replicates. For each of A, B, C clones and the control, three independent

6 cell cultures were included as dish-replicates. These 12 samples were randomized, processed,

7 and measured by a two-hour DIA-MS on Orbitrap Lumos. Quantification was preformed based

8 on high-resolute fragment signals (MS2 resolution, 30,000), followed by bioinformatic analysis

9 (Figure 1).

11 To analyse the DIA-MS data, we used both spectrum-centric [37] (named directDIA in

12 Spectronaut, a method extracting pseudo- spectrum directly from DIA data without the need of

13 an assay library $[38,39]$ ) and peptide-centric (based on SWATH assay library which contains

14 mass spectrometric assays for 10,000 human proteins [40], named panHuman hereafter)

15 approaches. Both approaches achieved substantial sensitivity when both peptide- and protein-

16 FDR were strictly controlled at $1 \%$ (Figure 2 A-B) [25]. On average, directDIA identified and

17 quantified 5,465 \pm 7 Swiss-Prot proteins, assigned from 61,476 \pm 430 unique peptides $(\sim 82,840$

$18 \pm 685$ peptide precursors) in the 12 samples, whereas panHuman library detected $5625 \pm 27$

19 proteins corresponding to $57,920 \pm 1,601$ unique peptides $(\sim 68,338 \pm 2,092$ peptide precursors $)$.

20 As expected, the majority of the proteins identified (i.e., 4,949 proteins) were shared between

21 directDIA and panHuman (Figure 2C). Interestingly, despite directDIA quantified 2.83\% less

22 proteins, this library-free approach profiled 6.14\% more peptides than panHuman library based

23 analysis. This could be partially explained by the fact that the panHuman library was originally

24 derived from TripleTOF platform [40], but still indicates the decent proteomic recovery,

25 essentially at the peptide level, achieved by directDIA. Impressively, directDIA reaches 98.9\%

26 of data completeness in measuring all the precursors (Supplementary Figure 1). Taken

27 together, we quantified 6,206 proteins in the combined results from the single measurements of

28 two hours, which represents $50-60 \%$ of the total proteome expressed in a cancer cell line under a

29 given condition [41]. 
1 We next focused on analyzing the quantitative reproducibility and variation in both clone- and 2 dish-replicates. Firstly, we found that 5487, 5277, 4361, and 5501 proteins of Control, Clone A, $3 \mathrm{~B}$, and $\mathrm{C}$ were quantified with a $\mathrm{CV}<20 \%$ in the three dish-replicates. The sample-to-sample

4 Pearson correlations were fairly high throughout the whole experiment (Figure 2D), with e.g., 5 average $\mathrm{R}=0.982$ achieved between dish-replicates in Controls across five orders of magnitude of absolute DIA MS2 intensity (see Supplementary Figure 2 for an example). Secondly, we performed both hierarchical clustering analysis (HCA) and principal component analysis (PCA)

8 on the relative quantification results (Figure 2 E-F). In both plots, we found the three dish9 replicates of Control and Clone $\mathrm{C}$ could emerge as individual clusters whereas Clone A and B 10 dish-replicates were not separated from each other. The fact that Clone $\mathrm{C}$ showed deviation from

11 to $\mathrm{A}$ and $\mathrm{B}$ could suggest a bigger clonal effect in $\mathrm{C}$ that was successfully revealed by DIA-MS

12 (Figure 2F). In the meanwhile, Clone $\mathrm{C}$ has similar growth and proliferation rate to $\mathrm{A}$ and $\mathrm{B}$

13 (Supplementary Figure 3). Consistent to PCA result, the average CV for Clone B dish-

14 replicates was $17.4 \%$, which was higher than that of other clones (i.e, $11.41 \%, 13.21 \%$, and

15 10.13\% for Control, Clone A and C respectively, Supplementary Figure 4), suggesting a bigger

16 dish-variation in B. Finally, all the 12 samples had an average CV of $21.56 \%$, higher than that of

17 each clones. Also, PCA plot indicates Control samples were separated from all knockout samples 18 using the first principal component, suggesting the proteomic variation due to HMGN1 depletion 19 was successfully captured in our whole data set. Overall, DIA-MS yielded excellent

20 quantification reproducibility, providing an opportunity to faithfully reveal the de facto dish-and 21 clone-variations, and to reveal HMGN1 dosage associated proteotype changes.

23 To uncover differentially expressed proteins between control and knockout clones, we performed 24 statistical analysis visualized by volcano plots (Figure 3). A total of 162, 123, and 297 proteins 25 were filtered as significantly regulated in Control vs. A, Control vs. B, and Control vs. C 26 comparisons, by using $\mathrm{BH}$ adjusted $\mathrm{P}$ value $<0.05$ and fold-change $>2$ as the criteria.

27 Remarkably, HMGN1 was identified by six unique peptides and indeed was the protein that 28 showed the largest fold-change in all of the three comparisons, with 83.42-, 67.14-, and 62.63-

29 folds decreased from control to a quasi- noise level after CRISPR deletion. Of note, even in 30 control samples, the HMGN1 protein intensity ranks below No. 3100, indicating that a less 
1 sensitive MS method quantifying e.g., $~ 3000$ proteins will likely to fail in the precise

2 quantification of HMGN1.

4 The quantification performance of DIA-MS on other differential proteins following HMGN1

5 depletion was visualized using extracted ion chromatographic peaks (XIC graphics, Figure 4A-

6 D as examples). In such a plot, both the isotopic MS1 features for the peptide precursor (lower

7 panel in XIC graphics) and the most intensive MS2 transitions whose number were specified

8 during directDIA (upper panel in XIC graphics) could be aligned and compared across all

9 samples. In the tryptic peptide examples representing for 60S ribosomal protein L22-like 1

10 (Figure 4A, protein level Control/CRISPR = 32.12 folds), Sterol O-acyltransferase 1 (Figure

11 4B, Control/CRISPR = 3.05 folds), Guanine deaminase (Figure 4C, Control/CRISPR $=0.365$

12 fold), and Procollagen C-endopeptidase enhancer 2 (Figure 4D, Control/CRISPR = 0.150 fold),

13 MS2- level quantification by DIA-MS was accurate and reproducible. A total of 44 differential

14 proteins were profiled as significant in all the three comparisons, and harbored the consistent up-

15 or down- regulations (Figure 4E-F). This indicates a reproducible proteomic perturbation after

16 HMGN1 knockout. To benefit from the inclusion of dish- and clone-replicates, we then applied

17 identical statistical criteria ( $\mathrm{BH}$ adjusted $\mathrm{P}$ value $<0.05$ and fold-change $>2$ ) to the comparison

18 between control $(n=3)$ and all the knockout samples $(n=9)$. Accordingly, 147 differential proteins

19 were filtered for following up bioinformatic analysis.

21 To understand the biological consequences after HMGN1 deletion, we performed the enrichment

22 analysis of GO and KEGG annotations on 147 proteins, taking the 6206 identified protein list as

23 background (Figure 5A). We found that GO biological processes (BP) such as Cell

24 differentiation (GO:0030154, $\mathrm{P}=7.52 \mathrm{E}-06$ ), Regulation of cell proliferation (GO:0008285,

$25 \mathrm{P}=0.0041)$, Receptor-mediated endocytosis (GO:0006898, $\mathrm{P}=1.07 \mathrm{E}-04)$, Negative regulation of

26 endopeptidase activity (GO:001095, $\mathrm{P}=0.0092)$, and Proteolysis (GO:0006508, $\mathrm{P}=0.0292$ ) were

27 significantly enriched in the altered proteome, indicating the cell fate and endocytosis related

28 processes were disturbed or remodeled. Many proteins annotated in "Negative regulation of cell

29 proliferation" were found to be downregulated after HMGN1 deletion (Figure 5A), which is

30 consistent to the mild but significant increase of cell proliferation observed in knockouts

31 (Supplementary Figure 3). In agreement to the above BPs, cellular components (CC) such as 
1 Extracellular exosome (GO:0070062, $\mathrm{P}=4.79 \mathrm{E}-04)$, Integral component of plasma membrane

2 (GO:0005887, $\mathrm{P}=0.0050)$, and Clathrin-coated vesicle ( $\mathrm{GO}: 0030136, \mathrm{P}=0.0022)$ associated

3 proteins were differentially regulated. Two KEGG pathways, Complement and coagulation

4 cascades $(\mathrm{P}=5.72 \mathrm{E}-4)$ and ECM-receptor interaction $(\mathrm{P}=0.0343)$, were also enriched in the 147-

5 protein list, reinforcing the newly discovered role of HMGN1 in immune response [33-36].

To extend functional analysis to all the quantified proteins in a threshold-free manner, we applied gene set enrichment analysis (GSEA) in all HMGN1 deleted cells compared to wildtype controls. GSEA uncovered many biological regulations (Figure 5B, FDR $q$-values all below

10 0.05). In control samples, we found that the Peroxisome, Ribosome and Cholesterol biosynthesis

11 functions were upregulated, suggesting these functions are impaired when HMGN1 is removed.

12 In comparison, many more gene sets were enriched in knockout phenotypes, which could be

13 summarized to a) Immune pathways, such as complement cascade and interferon alpha/ gamma

14 responses, b) Cancer signaling, such as Rig I or Toll like receptor, TNFa, and Kras signaling, as

15 well as angiogenesis, and c) Stress pathways, such as Reactive oxygen species pathway, hypoxia,

16 and apoptosis. Figure 5C and 5D shows significant enrichment examples of Complement and

17 coagulation cascades (KEGG) and Stress pathways (Biocarta) among upregulated processed after

18 HMGN1 deletion. In particular, we found that among the H3K27-related datasets in the C2 CGP

19 collection of MSigDB (Broad Institute) those genes marked by H3K27me3 (inactive histone

20 marks) in reprogrammed iPS cells (MIKKELSEN_MCV6_HCP_WITH_H3K27ME3) were

21 enriched in the HMGN1 knockout condition (FDR $=0.0021$, Figure 5E), confirming the deletion

22 of HMGN1 inactivates histone and chromatin activity [42]. Altogether, GSEA analysis helped in

23 the further dissection of proteotypes induced by HMGN1 deletion.

25 Finally, as a global confirmation of the relative quantification results, we measured the same

26 sample set by SWATH-MS method which is well established. Using panHuman library

27 SWATH-MS profiled 3767 proteins (protein- FDR 1\%). Indeed, the quantitative fold-changes

28 derived from directDIA analysis in both SWATH and DIA were nicely correlated (Pearson R

$29=0.863$, Figure 5F), suggesting that both measurements accurately quantified protein regulations

30 following HMGN1 deletion. 


\section{Discussion}

2 Our proteomic results shed the light on the cellular functions of HMGN1 protein. Firstly, as an

3 abundant member, HMGN1 belongs to HMGNs, a family of proteins which binds specifically to

4 the nucleosome core particle and reduces the compaction of the chromatin fiber [28]. HMGN1

5 has been discovered to modulate Histone $\mathrm{H} 3$ phosphorylation [28] and to remodel the chromatin

6 during embryogenesis [43]. In this context, this protein was found to preferentially localize to

7 DNase I hypersensitive sites, promoters, functional enhancers, and transcription factor binding

8 sites [30] and has a variant-specific and tissue-specific effect on global transcription [31,32].

9 Consistent to this major role of HMGN1 in regulating gene expression and DNA repair in

10 mammalians [42], we found that those genes marked by H3K27me3 in reprogrammed iPS cells

11 were enriched in knockout phenotypes using our proteomic data [44]. Even more interestingly,

12 the same gene set was found to be enriched in wildtype cells when compared to HMGN1

13 overexpressed B cell progenitors, according to a recent transcriptome analysis [27]. Taking these

14 results together, we can deduce that HMGN1 dosage, whether of overexpression, wildtype, and

15 depletion, has a linear relationship with histone activity in regulating the overall gene expression.

17 Secondly, in previous reports, the $\mathrm{Hmgn}^{-/-}$mice were found to have reduced fertility, higher

18 sensitivity to UV irradiation [29] and earlier signs of N-Nitrosodiethylamine-Induced liver

19 tumorigenesis than their wildtype littermates [45]. Loss of HMGN variants affects the expression

20 of stress-responsive genes in mouse embryonic fibroblasts (MEFs) [46]. We also found those

21 stress pathways including hypoxia and apoptosis are induced after HMGN1 knockout, potentially

22 explaining the vulnerability phenotypes discovered in $\mathrm{Hmgn}^{-/-}$mice.

24 Thirdly, the interaction of HMGN1 with chromatin was previously found to down-regulate the

25 expression of N-cadherin, a transmembrane protein mediating cell-cell adhesion [47]. Notably,

26 we detected significant proteomic changes (both up- and down- regulations) in the extracellular

27 exosome and plasma membrane sub-proteomes, which might suggest that the "outside" proteome

28 was already heavily rewired as a new state of HMGN1 deletion in the tested cell line. Further

29 studies are therefore needed to delineate the relationship between HMGN1 downregulation and

30 extracellular protein changes.

31

32 Last but not the least, HMGN1 recently was re-discovered to play an important role in immune

33 response. It was identified as an alarmin, a group of mediators capable of promoting the 
1 recruitment and activation of antigen-presenting cells (APCs), including dendritic cells (DCs),

2 that can potentially alert host defense against danger signals. Yang et al reported that

3 extracellular HMGN1 acts as a novel alarmin that binds TLR4 and induces antigen-specific Th1

4 immune responses [33] and could be a potent immunoadjuvant by supporting $\mathrm{T}$ cell-mediated

5 antitumor immunity $[34,35]$. Recently a curative therapeutic vaccination regimen dubbed

6 'TheraVac' consisting of HMGN1 and resiquimod plus a checkpoint inhibitor was developed

7 with the potential to eliminate various large tumors and induce tumor-specific immunity [36].

8 Our proteomic profiling is promising in this regard, because complement and coagulation

9 cascades and interferon alpha or gamma responses were found to be significantly induced upon

10 HMGN1 deletion, and therefore provides additional insights of HMGN1's role in immunity.

12 The specific clone- and dish-effects we observed could be derived from possible CRISPR off-

13 target effects, the real-time cell culturing difference, and/or experimental variations. There was

14 no apparent cell proliferation difference between knockout clones. It is conceivable that

15 proteotype provides closer scrutiny on phenotypes than mRNA profiling. Therefore, for a

16 proteomic measurement on CRISPR effect, we recommend multiple clone- and dish-replicates

17 to be included in the experiment to fully capture the proteotypic variations.

19 In summary, we applied a powerful DIA-MS measurement featuring decent sensitivity $(>6200$

20 proteins profiled), high reproducibility ( $\mathrm{R}>0.98$ between dish- replicates), high quantitative

21 accuracy (for both absolute and relative scale quantification), cost-effectiveness (label-free

22 quantification), and high sample throughput (2 hours per single measurement) in analyzing the

23 cellular proteome after HMGN1 CRISPR deletion. Both the targeted protein (i.e., HMGN1) and

24 the differential proteome closely associated to HMGN1 deletion were rapidly profiled. Our

25 results provide new hints in understanding HMGN1 functions, and suggest the DIA-MS can be

26 used as a rapid, robust, and reliable proteomic-screening step to assess the outcome of the

27 CRISPR experiments. 
1 Methods

2 Cell culture and transfection

3 T-REx-HeLa CCL2 cells were purchased from Invitrogen and cultured in DMEM (4.5 g/1

4 glucose, $2 \mathrm{mM} \mathrm{L-glutamin)} \mathrm{(Gibco)} \mathrm{supplemented} \mathrm{with} \mathrm{10 \%} \mathrm{fetal} \mathrm{bovine} \mathrm{serum} \mathrm{(BioConcept),}$

$5100 \mathrm{U} / \mathrm{ml}$ penicillin (Gibco) and $100 \mu \mathrm{g} / \mathrm{ml}$ streptomycin (Gibco) at $37^{\circ} \mathrm{C}$ in a humidified

6 incubator with 5\% CO2. DNA transfection of the cells was performed with jetPrime (Polyplus)

7 according to the manufactors's instructions. The cell pellets were washed by pre-cold PBS twice

8 and were snap frozen before proteomic sample preparation.

\section{MTT assay measuring cell proliferation}

11 T-REx-HeLa CCL2 cells and CRISPR/Cas9-engineered HMGN1 knockout clones were seeded

12 in 6-well plates and treated with $0.1 \mathrm{mg} / \mathrm{ml} \mathrm{MTT} \mathrm{(3-[4,5-dimethylthiazol-2-yl]-2,5-}$

13 diphenyltetrazolium bromide, Sigma) for $4 \mathrm{~h}$ at $37^{\circ} \mathrm{C}$. After removal of the media the converted

14 dye was solubilized in $80 \%$ isopropanol, $20 \%$ DMSO. The absorbance of the converted dye was

15 measured at a wavelength of $570 \mathrm{~nm}$ with background subtraction at $670 \mathrm{~nm}$ (Synergy HT,

16 BioTek).

\section{CRISPR/Cas9-mediated gene knockout}

19 CRISPR guideRNAs were designed based on their specificity score retrieved from the Optimized

20 CRISPR Design web tool (http:crispr.mit.edu). Annealed DNA oligonucleotides containing the

21 target sequence were cloned into the hSpCas9 plasmid (pX458, Addgene) using BbsI restriction

22 sites. T-REx-HeLa cells were transfected with two hspCas9 constructs encoding gRNAs that

23 target the second and third exon of the target gene HMGN1, respectively. The cell culture

24 medium was replaced 4 hours after transfection and cells were recovered for 72 hours. For FACS

25 sorting $1 \times 10 \mathrm{e} 6$ cells were gently detached from the tissue culture plate with $0.25 \%$ trypsin-

26 EDTA (Gibco) and resuspended in PBS containing 1\% FBS. GFP-positive cells were isolated by

27 FACS (BD Facs Aria IIIu sorter) and single cells were sorted into each well of a 96-well plate.

28 Cell clones were expanded for three weeks and then screened for deletion events by western-

29 blotting. Below lists the information of gRNA target sequences and oligonucleotides.

30 gRNA target sequences

31 gRNA_1_HMGN1_exon2: CCGCAGGTCAGCTCCGCCGAAGG 
gRNA_2_HMGN1_exon3: CGCCGATCTCCTCTTGGGCTTGG

2 Oligonucleotides

3 Hmgn1_gRNA_1_exon2_forward: 5-CACCGCCGCAGGTCAGCTCCGCCGA-3

4 Hmgn1_gRNA_1_exon2_reverse: 5- AAACTCGGCGGAGCTGACCTGCGGC-3

5 Hmgn1_gRNA_2_exon3_forward: 5-CACCGCGCCGATCTCCTCTTGGGCT-3

6 Hmgn1_gRNA_2_exon3_reverse: 5-AAACAGCCCAAGAGGAGATCGGCGC-3

8 Western-Blotting

9 For immunoblot analysis 1x10e6 T-REx-HeLa cells were lysed in $100 \mu 1$ lysis buffer $(0.5 \%$

$10 \mathrm{NP} 40,50 \mathrm{mM}$ Tris- $\mathrm{HCl}, \mathrm{pH}$ 8.0, $150 \mathrm{mM} \mathrm{NaCl}, 50 \mathrm{mM} \mathrm{NaF}$ supplemented with $1 \mathrm{mM}$ PMSF

11 and protease inhibitors (Sigma)). The cell lysate was cleared by centrifugation (15000xg, 20

$12 \mathrm{~min}$ ) and boiled for $5 \mathrm{~min}$ after addition of $3 \mathrm{x}$ Laemmli sample buffer. The denatured sample

13 was loaded on NuPAGE 4-12\% Bis-Tris SDS-PAGE gels (Invitrogen) for gel electrophoresis

14 and then transferred onto nitrocellulose membranes (Trans-Blot Turbo, BioRad). Proteins were

15 detected by rabbit polyclonal antibodies against HMGN1 (\#5692, Cell Signaling) and mouse

16 monoclonal antibodies against Actin (ab179467, Abcam). For immunodetection by enhanced

17 chemiluminescence (ECL, Amersham) horseradish-peroxidase-coupled secondary antibodies

18 (\#7074, Cell Signaling and \#115035003, Jackson ImmunoResearch) were used.

\section{Cell lysis and in-solution digestion}

21 The protein extraction and digestion were preformed as previously described [23]. Briefly, cell

22 pellets were suspended in $10 \mathrm{M}$ urea lysis buffer and complete protease inhibitor cocktail

23 (Roche), ultrasonically lysed at $4^{\circ} \mathrm{C}$ for 2 minutes by two rounds using a VialTweeter device

24 (Hielscher-Ultrasound Technology). The mixtures were centrifuged at 18,000 g for 1 hour to

25 remove the insoluble material. The supernatant protein amount was quantified by Bio-Rad 26 protein assay. Protein samples were reduced by $10 \mathrm{mM}$ Tris-(2-carboxyethyl)-phosphine for 1

27 hour at $37^{\circ} \mathrm{C}$ and $20 \mathrm{mM}$ iodoacetamide in the dark for 45 minutes at room temperature. All the 28 samples were further diluted by $1: 6(\mathrm{v} / \mathrm{v})$ with $100 \mathrm{mM} \mathrm{NH}_{4} \mathrm{HCO}_{3}$ and were digested with 29 sequencing-grade porcine trypsin (Promega) at a protease/protein ratio of $1: 25$ overnight at $37^{\circ} \mathrm{C}$.

30 The amount of the purified peptides was determined using Nanodrop ND-1000 (Thermo

31 Scientific) and $1.5 \mu \mathrm{g}$ peptides were injected in each LC-MS run. 
2 DIA mass spectrometry

3 The peptide separation was performed on EASY-nLC 1200 systems (Thermo Scientific) using a

4 self-packed analytical PicoFrit column (New Objective) (75 $\mu \mathrm{m}$ x $60 \mathrm{~cm}$ length). This $50 \mathrm{~cm}$

5 column was packed with ReproSil-Pur 120A C18-Q $1.9 \mu \mathrm{m}$ (Dr. Maisch GmbH). An LC method

6 of 2 hours was used (Buffer A: 0.1\% formic acid, buffer B: $80 \%$ acetonitrile containing $0.1 \%$

7 formic acid). Briefly, a 5\% to 37\% Buffer B gradient of 109-min was conducted with the flow

8 rate of $300 \mathrm{nl} / \mathrm{min}$ at $60{ }^{\circ} \mathrm{C}$ in a column oven (PRSO-V1, Sonation $\mathrm{GmbH}$ ).

10 The Orbitrap Fusion Lumos Tribrid mass spectrometer (Thermo Scientific) with a NanoFlex ion

11 source was coupled to the LC platform. Spray voltage was set to be 2,000 V and heating

12 capillary was kept at $275^{\circ} \mathrm{C}$. The DIA-MS method consisted of a MS1 survey scan and 40 MS2

13 scans of variable windows. The MS1 scan range is $350-1650 \mathrm{~m} / \mathrm{z}$ and the MS1 resolution is

$14120 \mathrm{k}$ at $\mathrm{m} / \mathrm{z} 200$. The MS1 full scan AGC target value was set to be 2.0E5 and the maximum

15 injection time was $100 \mathrm{~ms}$. The MS2 resolution was set to 30,000 at m/z 200 and normalized

16 HCD collision energy was $28 \%$. The MS2 AGC was set to be 5.0E5 and the maximum injection

17 time was $50 \mathrm{~ms}$. The default peptide charge state was set to 2. Both of MS1 and MS2 spectra

18 were recorded in profile mode.

19 The additional SWATH-MS measurements were performed as previously published [26].

\section{DIA data extraction and analysis}

22 The DIA runs were analysed with Spectronaut Pulsar version 12.0 (Biognosys AG, Switzerland)

$23[38,48]$. In the directDIA approach, DIA runs were directly searched against Swiss-Prot protein

24 database (March 2018, 20,258 entries) with following settings: full tryptic allowing two missed

25 cleavages, set carbamidomethylation as a fixed modification on all cysteines, set oxidation of

26 methionines, protein N-terminal acetylation as dynamic modifications. Both precursor and

27 protein FDR were controlled at 1\%. As for quantification, interference correction function was

28 enabled, and top 3 peptide precursors were summed for protein quantification. Data filtering was

29 done with Qvalue sparse in this 12-sample experiment. In panHuman approach, the published set

30 of mass spectrometric assays for 10,000 human proteins was downloaded [40] and the protein

31 quantitative results were summarized in the same way as directDIA. In both approaches, all the 
1 other parameters in Spectronaut are kept as default unless mentioned. To combine directDIA and

2 panHuman results, the protein quantification results of those only acquired by panHuman library

3 (but not by directDIA) were simply appended into the directDIA results.

4

5 Bioinformatic analysis

6 Hierarchical clustering analysis (HCA) was performed by Cluster 3.0 on the normalized, $\log 2-$

7 transformed, 2-dimensional-centered DIA-MS intensities and visualized by TreeView. Principal

8 component analysis (PCA) was performed by R software on the normalized, log2-transformed

9 data. Quantitative CVs were calculated by using normalized DIA data at the original scale. For

10 differential expression analysis, $P$ values were corrected for multiple testing with the Benjamini-

11 Hochberg (BH) method [49]. DAVID Bioinformatics Resources 6.8 (https://david.ncifcrf.gov/)

12 [50] was used to perform the enrichment analysis of biological processes, cellular component

13 and pathways in the 147-protein list. Representative proteins and their enriched items were

14 visualized by Cytoscape v3.70 [51]. GSEA 3.0 (Gene set enrichment analysis [44]) was

15 downloaded from http://software.broadinstitute.org/gsea/index.jsp, and default settings were

16 used to infer the degree to which a given gene set is overrepresented at the upper or lower ends

17 of a ranked list of genes by at an estimated probability (i.e., FDR-q value). Briefly, a running-

18 sum statistic is generated from the ranked list of genes, with the magnitude of the increment

19 depending on the correlation of the gene with the phenotype (control and HMGN1 deletion).

\section{Acknowledgement}

23 We thank Lukas Reiter and Roland Bruderer from Biognosys AG for the technical assistance.

24 We would like to particularly thank Dr. Ruedi Aebersold for the resource support and helpful

25 discussions. M.M. was supported by a Long-Term Fellowship from the European Molecular

26 Biology Organization (ALTF 928-2014). 
1 Figure legend

2 Figure 1. Experimental schema. Two gRNAs targeting the second and third exon of HMGN1 3 were used for the CRISPR/Cas9-mediated deletion. Three knockout clone- replicates and three

4 dish- replicates per each clone and control cells were processed and measured by DIA

5 proteomics, followed by bioinformatics.

7 Figure 2. Identification and quantification results by DIA-MS. (A) Distribution of the

8 number of peptide precursors identified in each sample. KO, knockout. (B) Distribution of non-

9 redundant Swiss-Prot protein groups in each sample. (C) Venn diagram of protein identities

10 identified by directDIA (library free) and panHuman library based approaches. (D) Sample-to-

11 sample Pearson correlation coefficient R visualized. (E) Hierarchical clustering analysis (HCA)

12 on median normalized, log2-transformed DIA-MS data. (F) Principal component analysis (PCA)

13 between 12 samples. PC, principal component.

Figure 3. Volcano plots for the differential protein profiling between control and knockout

16 clones using three dish- replicates. HMGN1 was highlighted by the yellow dot.

Figure 4. Reproducible and consistent DIA quantification on protein changes induced by

19 HMGN1 knockout. (A) Extracted ion chromatography (XIC graphics) for TGNLGNVVHIER

20 (Charge 3+) of RPL22L1 (Swiss-Prot ID: Q6P5R6) between 12 samples. (B) XIC graphics for

21 NPTFLDYVRPR (Charge 2+) of SOAT1 (P35610). (C) XIC graphics for

22 EWC[+57]FKPC[+57]EIR (Charge 3+) of GDA (Q9Y2T3). (D) XIC graphics for

23 YC[+57]GDSPPAPIVSER (Charge 2+) of PCOLCE2 (Q9UKZ9). (E) Venn diagram of

24 significant protein identities based on Clone A, B, and C. (F) Fold-changes of 44 overlapping,

25 significantly regulated proteins in three clones. (G) Venn diagram of significant protein identities

26 as (E). The grey circle denotes Control samples compared to Clone A, B, C combined as a

27 knockout $(\mathrm{KO})$ group, representing 147 proteins.

29 Figure 5. Annotation and enrichment analysis of proteome regulated after HMGN1

30 deletion. (A) Representative GO biological processes and KEGG pathways enriched in 147

31 protein list, and proteins annotated in each item. (B) GSEA analysis towards the full quantitative 
1 data set of all proteins using gene sets from KEGG, Reactome and HALLMARK summary. (C-

2 E) Gene set examples enriched in KO group. (F) Foldchange correlation between SWATH-MS and DIA-MS based on directDIA approach.

\section{References}

[1] Barrangou, R., The roles of CRISPR-Cas systems in adaptive immunity and beyond. Curr

13 [2] Hsu, P. D., Lander, E. S., Zhang, F., Development and applications of CRISPR-Cas9 for genome engineering. Cell 2014, 157, 1262-1278.

[3] Cong, L., Ran, F. A., Cox, D., Lin, S., et al., Multiplex genome engineering using CRISPR/Cas systems. Science 2013, 339, 819-823.

[4] Mali, P., Yang, L., Esvelt, K. M., Aach, J., et al., RNA-guided human genome engineering via Cas9. Science 2013, 339, 823-826.

[5] Jinek, M., Chylinski, K., Fonfara, I., Hauer, M., et al., A programmable dual-RNA-guided DNA endonuclease in adaptive bacterial immunity. Science 2012, 337, 816-821.

[6] Fonfara, I., Richter, H., Bratovic, M., Le Rhun, A., Charpentier, E., The CRISPR-associated DNA-cleaving enzyme Cpf1 also processes precursor CRISPR RNA. Nature 2016, 532, 517-

23521.

24 [7] Lin, J., Wong, K. C., Off-target predictions in CRISPR-Cas9 gene editing using deep 25 learning. Bioinformatics 2018, 34, i656-i663.

[8] Kim, D., Kim, J. S., DIG-seq: a genome-wide CRISPR off-target profiling method using chromatin DNA. Genome research 2018.

[9] Lazzarotto, C. R., Nguyen, N. T., Tang, X., Malagon-Lopez, J., et al., Defining CRISPR-Cas9 genome-wide nuclease activities with CIRCLE-seq. Nature protocols 2018, 13, 2615-2642. [10] Akcakaya, P., Bobbin, M. L., Guo, J. A., Malagon-Lopez, J., et al., In vivo CRISPR editing with no detectable genome-wide off-target mutations. Nature 2018, 561, 416-419. [11] Ran, F. A., Hsu, P. D., Lin, C. Y., Gootenberg, J. S., et al., Double nicking by RNA-guided CRISPR Cas9 for enhanced genome editing specificity. Cell 2013, 154, 1380-1389.

34 [12] Ran, F. A., Hsu, P. D., Wright, J., Agarwala, V., et al., Genome engineering using the CRISPR-Cas9 system. Nature protocols 2013, 8, 2281-2308. Cas9 off-target effects in human cells. Nature methods 2015, 12, 237-243, 231 p following 243.

40 Analysis of CRISPR-Cas Complexes on Next Generation Sequencing Chips. Cell 2017, 170, $41 \quad 35-47$ e13. 
[15] Cameron, P., Fuller, C. K., Donohoue, P. D., Jones, B. N., et al., Mapping the genomic landscape of CRISPR-Cas9 cleavage. Nature methods 2017, 14, 600-606.

[16] Tsai, S. Q., Nguyen, N. T., Malagon-Lopez, J., Topkar, V. V., et al., CIRCLE-seq: a highly sensitive in vitro screen for genome-wide CRISPR-Cas9 nuclease off-targets. Nature methods 2017, 14, 607-614.

[17] Aebersold, R., Burlingame, A. L., Bradshaw, R. A., Western blots versus selected reaction monitoring assays: time to turn the tables? Molecular \& cellular proteomics : MCP 2013, 12, 2381-2382.

[18] Picotti, P., Aebersold, R., Selected reaction monitoring-based proteomics: workflows, potential, pitfalls and future directions. Nature methods 2012, 9, 555-566.

[19] Aebersold, R., Mann, M., Mass spectrometry-based proteomics. Nature 2003, 422, 198207.

[20] Aebersold, R., Mann, M., Mass-spectrometric exploration of proteome structure and function. Nature 2016, 537, 347-355. [21] Gillet, L. C., Navarro, P., Tate, S., Rost, H., et al., Targeted data extraction of the MS/MS spectra generated by data-independent acquisition: a new concept for consistent and accurate proteome analysis. Molecular \& cellular proteomics : MCP 2012, 11, 0111016717. [22] Liu, Y., Buil, A., Collins, B. C., Gillet, L. C., et al., Quantitative variability of 342 plasma proteins in a human twin population. Molecular systems biology 2015, 11, 786. [23] Collins, B. C., Hunter, C. L., Liu, Y., Schilling, B., et al., Multi-laboratory assessment of reproducibility, qualitative and quantitative performance of SWATH-mass spectrometry. Nature communications 2017, 8, 291. [24] Rost, H. L., Rosenberger, G., Navarro, P., Gillet, L., et al., OpenSWATH enables automated, targeted analysis of data-independent acquisition MS data. Nat Biotech 2014, $32,219-223$. [25] Rosenberger, G., Bludau, I., Schmitt, U., Heusel, M., et al., Statistical control of peptide and protein error rates in large-scale targeted data-independent acquisition analyses. Nature methods 2017, 14, 921-927.

[26] Liu, Y., Borel, C., Li, L., Muller, T., et al., Systematic proteome and proteostasis profiling in human Trisomy 21 fibroblast cells. Nature communications 2017, 8, 1212.

[27] Mowery, C. T., Reyes, J. M., Cabal-Hierro, L., Higby, K. J., et al., Trisomy of a Down Syndrome Critical Region Globally Amplifies Transcription via HMGN1 Overexpression. Cell reports 2018, 25, 1898-1911 e1895. [28] Lim, J. H., Catez, F., Birger, Y., West, K. L., et al., Chromosomal protein HMGN1 modulates histone H3 phosphorylation. Molecular cell 2004, 15, 573-584. enhances the rate of DNA repair in chromatin. EMBO J 2003, 22, 1665-1675. [30] Cuddapah, S., Schones, D. E., Cui, K., Roh, T. Y., et al., Genomic profiling of HMGN1 reveals an association with chromatin at regulatory regions. Mol Cell Biol 2011, 31, 70040709.

41 [31] Rochman, M., Taher, L., Kurahashi, T., Cherukuri, S., et al., Effects of HMGN variants on the cellular transcription profile. Nucleic Acids Res 2011, 39, 4076-4087.

44 [32] Kugler, J. E., Horsch, M., Huang, D., Furusawa, T., et al., High mobility group N proteins modulate the fidelity of the cellular transcriptional profile in a tissue- and variant-specific 45 manner.J Biol Chem 2013, 288, 16690-16703. 
[33] Yang, D., Postnikov, Y. V., Li, Y., Tewary, P., et al., High-mobility group nucleosomebinding protein 1 acts as an alarmin and is critical for lipopolysaccharide-induced immune responses. J Exp Med 2012, 209, 157-171.

[34] Wei, F., Yang, D., Tewary, P., Li, Y., et al., The Alarmin HMGN1 contributes to antitumor immunity and is a potent immunoadjuvant. Cancer Res 2014, 74, 5989-5998.

[35] Nie, Y., Yang, D., Oppenheim, J. J., Alarmins and Antitumor Immunity. Clin Ther 2016, 38, 1042-1053.

[36] Nie, Y., Yang, Trivett, A., Han, Z., et al., Development of a Curative Therapeutic Vaccine (TheraVac) for the Treatment of Large Established Tumors. Sci Rep 2017, 7, 14186. [37] Ting, Y. S., Egertson, J. D., Payne, S. H., Kim, S., et al., Peptide-Centric Proteome Analysis: An Alternative Strategy for the Analysis of Tandem Mass Spectrometry Data. Molecular \& cellular proteomics : MCP 2015, 14, 2301-2307.

[38] Bruderer, R., Bernhardt, O. M., Gandhi, T., Xuan, Y., et al., Optimization of Experimental Parameters in Data-Independent Mass Spectrometry Significantly Increases Depth and Reproducibility of Results. Molecular \& cellular proteomics : MCP 2017, 16, 2296-2309. [39] Novy, K., Kilcher, S., Omasits, U., Bleck, C. K. E., et al., Proteotype profiling unmasks a viral signalling network essential for poxvirus assembly and transcriptional competence. Nat Microbiol 2018, 3, 588-599. [40] Rosenberger, G., Koh, C. C., Guo, T., Rost, H. L., et al., A repository of assays to quantify 10,000 human proteins by SWATH-MS. Scientific data 2014, 1, 140031. [41] Beck, M., Schmidt, A., Malmstroem, J., Claassen, M., et al., The quantitative proteome of a human cell line. Molecular systems biology 2011, 7, 549.

24 [42] Reeves, R., High mobility group (HMG) proteins: Modulators of chromatin structure and DNA repair in mammalian cells. DNA Repair (Amst) 2015, 36, 122-136.

[43] Furusawa, T., Lim, J. H., Catez, F., Birger, Y., et al., Down-regulation of nucleosomal binding protein HMGN1 expression during embryogenesis modulates Sox9 expression in chondrocytes. Mol Cell Biol 2006, 26, 592-604. analysis: a knowledge-based approach for interpreting genome-wide expression profiles. Proceedings of the National Academy of Sciences of the United States of America 2005, 102, 15545-15550. nucleosome-binding protein HMGN1 affects the rate of N-nitrosodiethylamine-induced hepatocarcinogenesis in mice. Mol Cancer Res 2014, 12, 82-90. [46] Deng, T., Zhu, Z. I., Zhang, S., Postnikov, Y., et al., Functional compensation among HMGN variants modulates the DNase I hypersensitive sites at enhancers. Genome research 2015, 25, 1295-1308. HMGN1 modulates the expression of N-cadherin. FEBS J 2005, 272, 5853-5863. [48] Bruderer, R., Bernhardt, O. M., Gandhi, T., Miladinovic, S. M., et al., Extending the limits of quantitative proteome profiling with data-independent acquisition and application to acetaminophen-treated three-dimensional liver microtissues. Molecular \& cellular proteomics : MCP 2015, 14, 1400-1410.

[49] Benjamini, Y., Hochberg, Y., Controlling the False Discovery Rate: A Practical and Powerful Approach to Multiple Testing. Journal of the Royal Statistical Society. Series B (Methodological) 1995, 57, 289-300. 
1 [50] Huang da, W., Sherman, B. T., Lempicki, R. A., Systematic and integrative analysis of 2 large gene lists using DAVID bioinformatics resources. Nature protocols 2009, 4, 44-57.

3 [51] Shannon, P., Markiel, A., Ozier, O., Baliga, N. S., et al., Cytoscape: a software

4 environment for integrated models of biomolecular interaction networks. Genome research

$52003,13,2498-2504$.

6 [52] Vizcaino, J. A., Csordas, A., del-Toro, N., Dianes, J. A., et al., 2016 update of the PRIDE

7 database and its related tools. Nucleic Acids Res 2016, 44, D447-456. 


\section{Figure 1. Experimental schema.}

\section{CRISPR experiments}
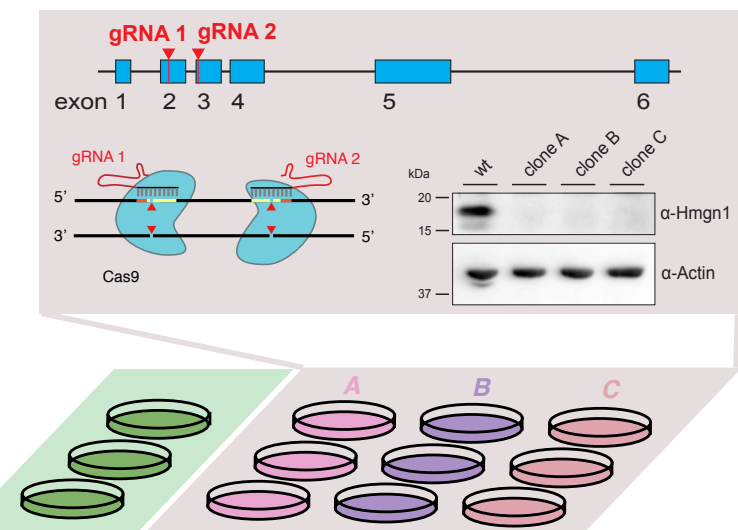

DIA mass spectrometry
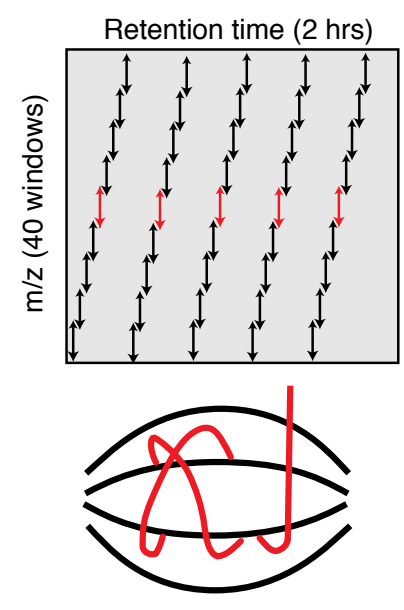

Orbitrap Lumos
Bioinformatic analysis

D 7000 Lproteins $=6206($ protein-FDR 1\%)

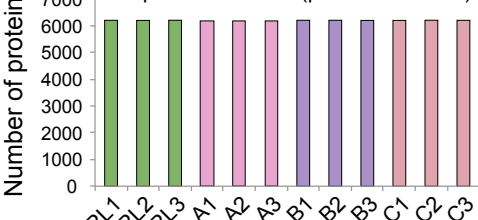

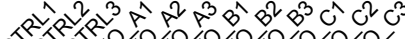

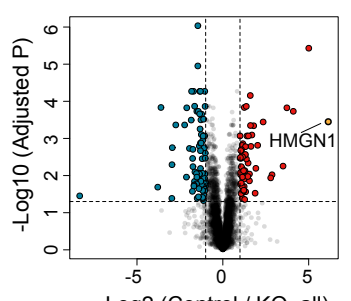

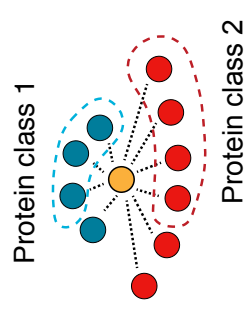


Figure 2. Identification and quantification results by DIA-MS.

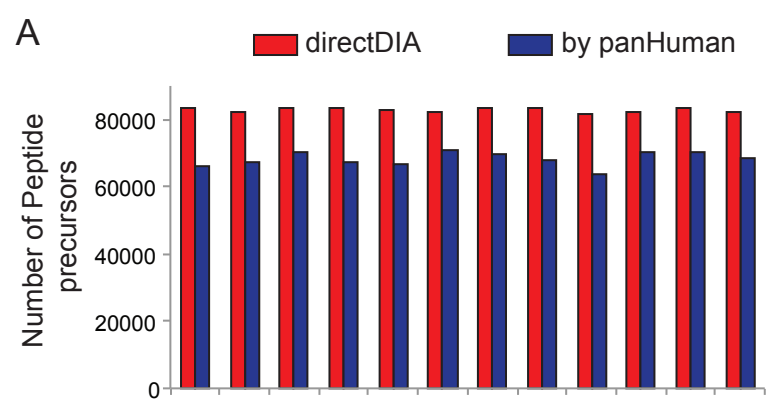

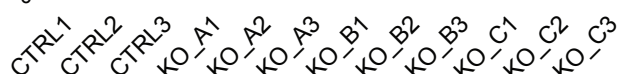

B

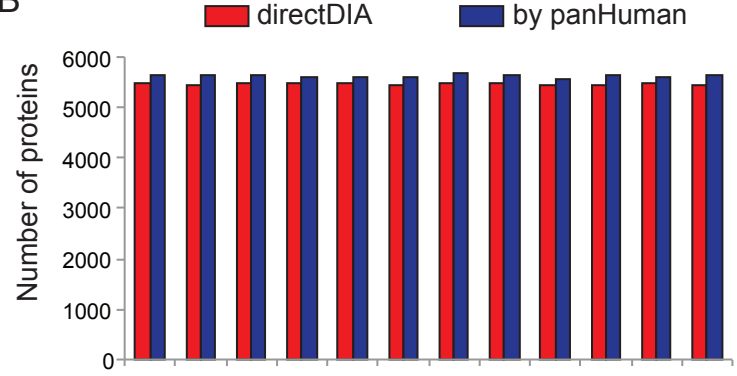

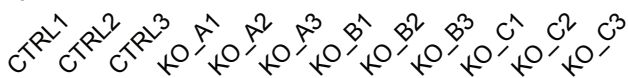

C

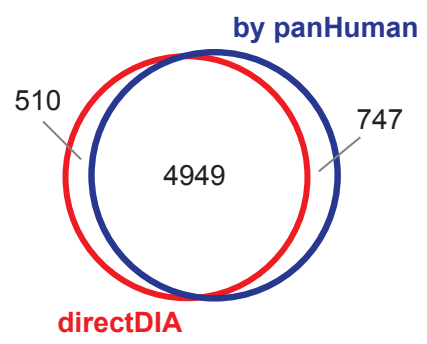

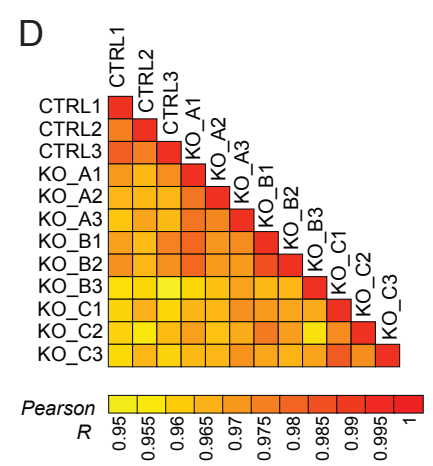

E

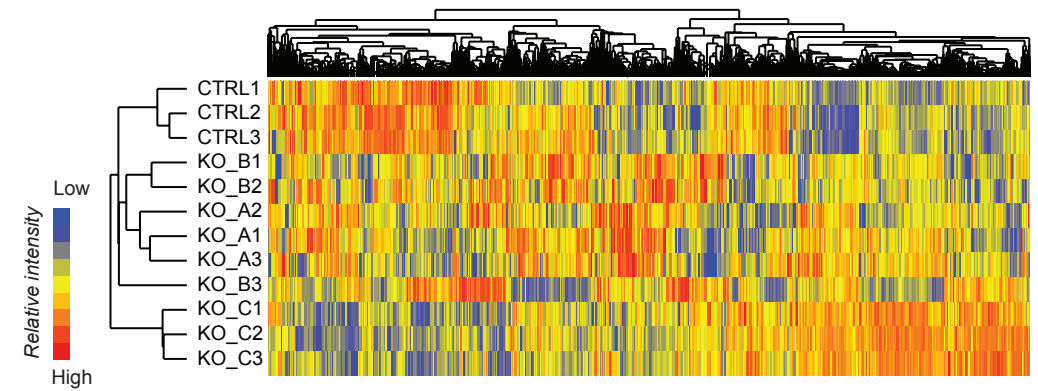

F

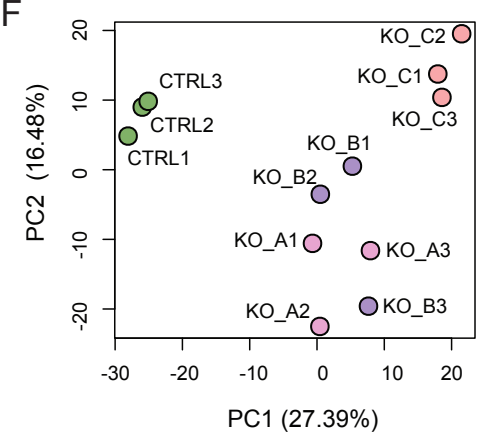


Figure 4. Reproducible and consistent DIA quantification on protein changes.
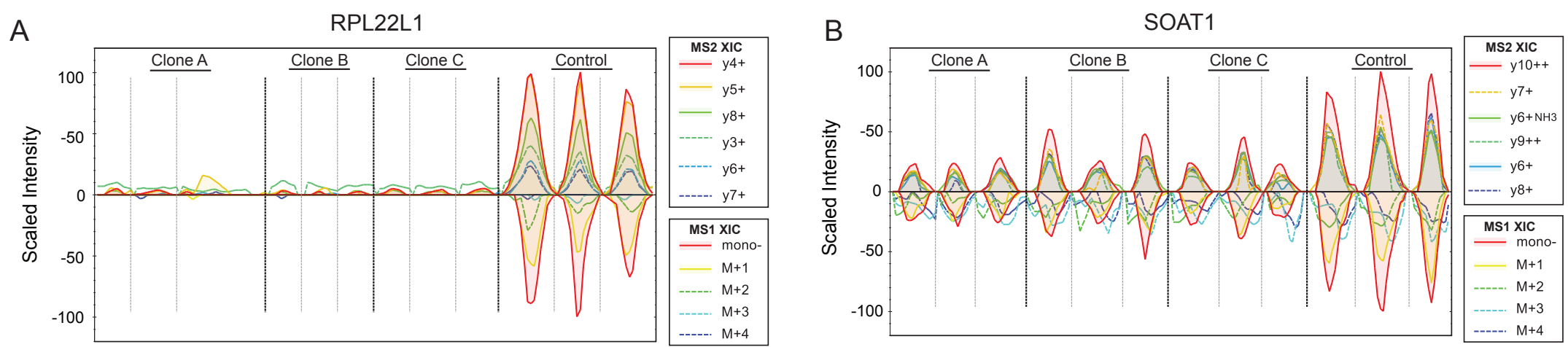

C
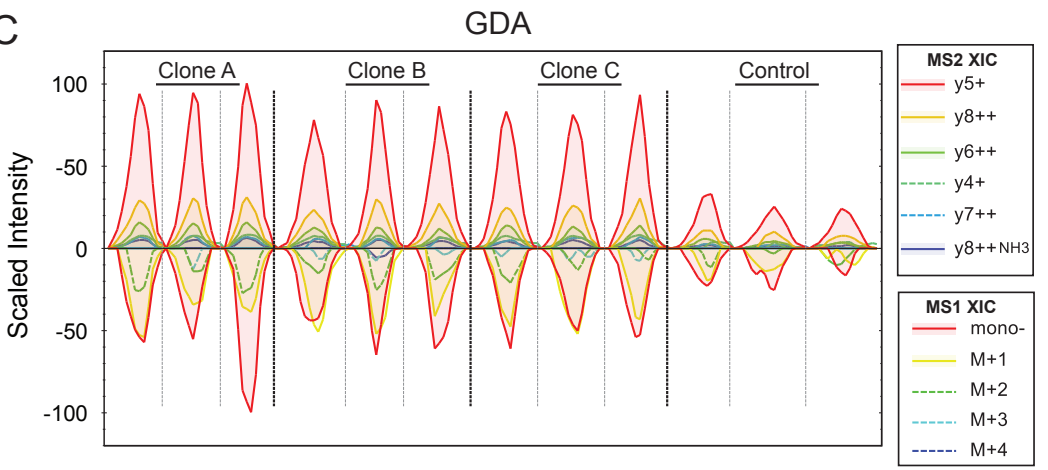

D
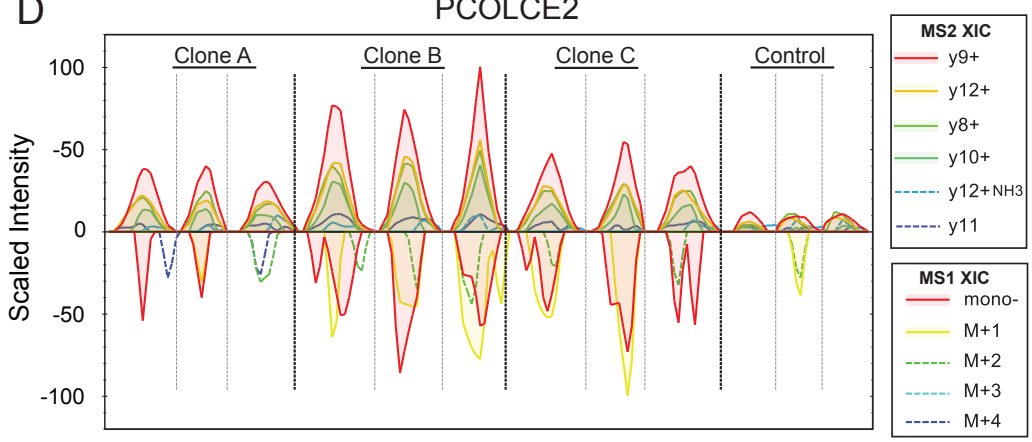

E

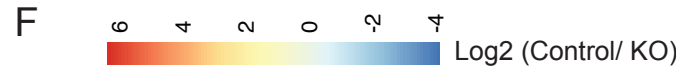

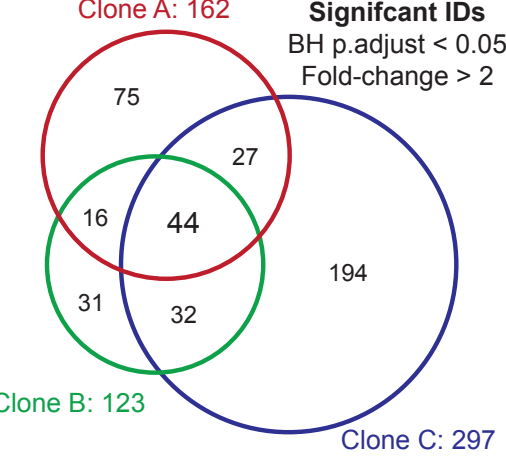

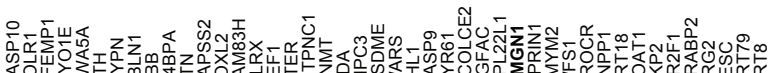

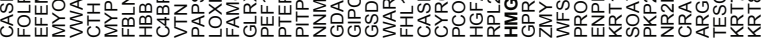

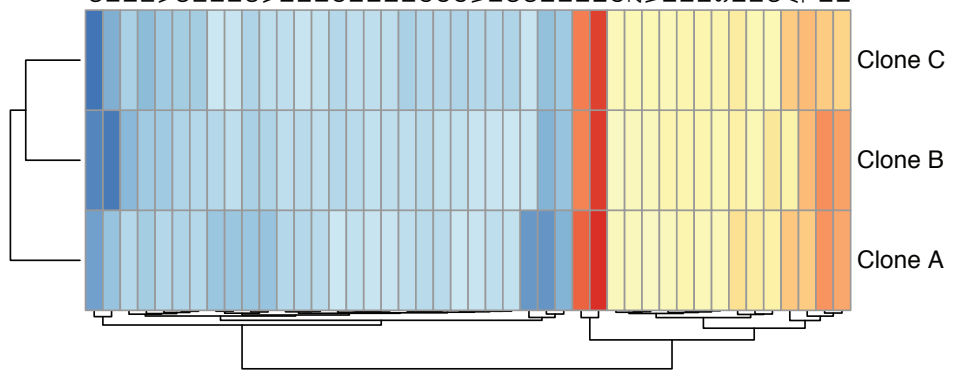

G

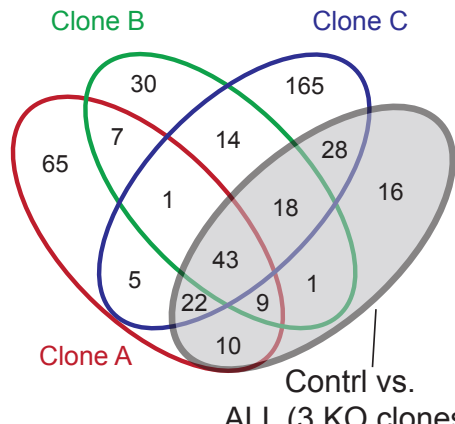

ALL (3 KO clones) 147 proteins 


\section{Figure 5. Annotation and enrichment analysis of proteome regulated after HMGN1 deletion.}

A

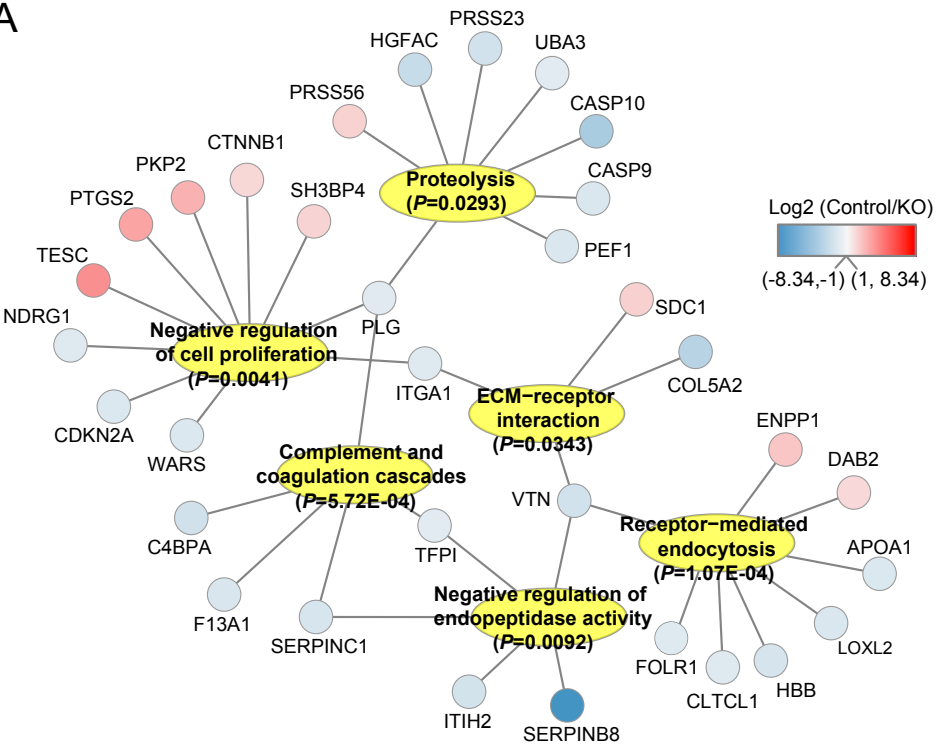

B

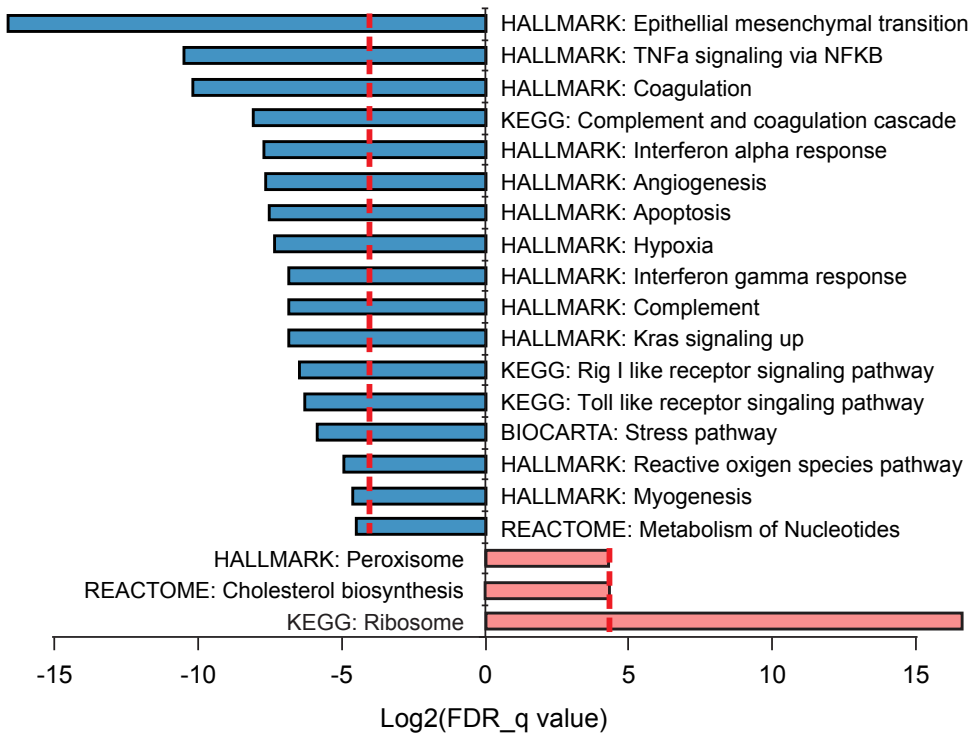

C

KEGG: Complement and coagulation cascade

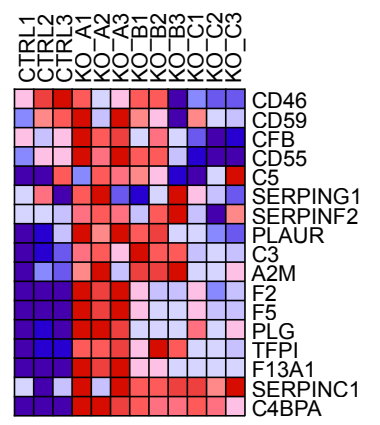

D

BIOCARTA: Stress Pathway

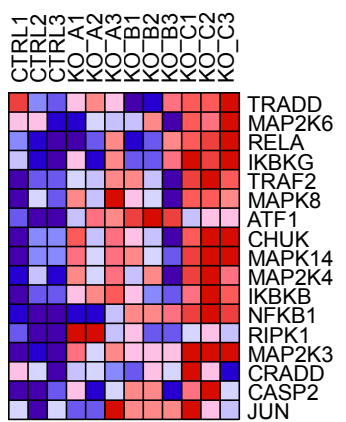

E MIKKELSEN_MEF_LCP_ WITH_H'̈K4MË3

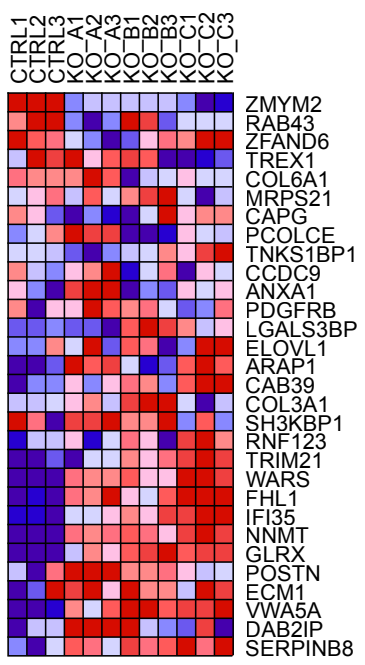

$\mathrm{F}$

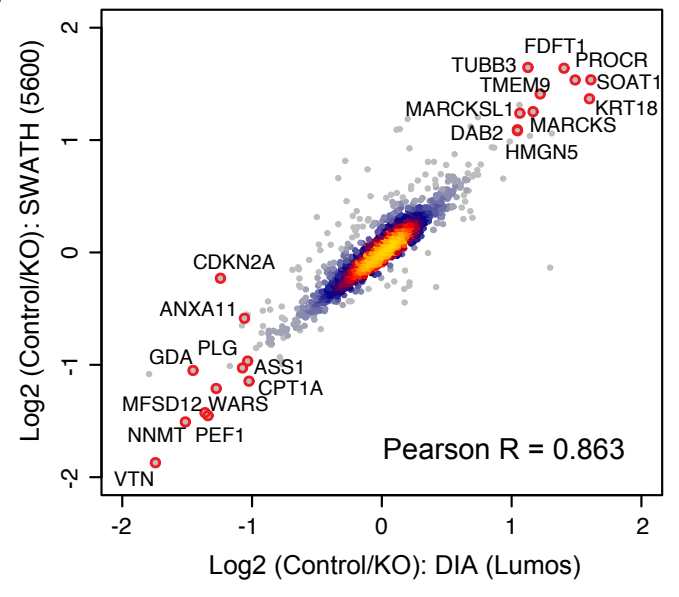

